\title{
Cultivable bacterial diversity of the canine dental plaque as a potential source of bacterial infections
}

\author{
Marián Mad’ar ${ }^{1}$, Jana Kačírová1, Aladár Mad'ari², Rastislav Mucha3 ${ }^{3}$ Eva Styková4, \\ Radomíra Nemcová ${ }^{1}$
}

${ }^{1}$ University of Veterinary Medicine and Pharmacy in Košice, Department of Microbiology and Immunology, Košice, Slovak Republic

${ }^{2}$ University of Veterinary Medicine and Pharmacy in Košice, University Veterinary Hospital, Košice, Slovak Republic

${ }^{3}$ Slovak Academy of Sciences, Biomedical Research Center, Institute of Neurobiology, Košice, Slovak Republic

${ }^{4}$ University of Veterinary Medicine and Pharmacy in Košice, Clinic of Horses, Košice, Slovak Republic

Received October 5, 2020

Accepted May 26, 2021

\begin{abstract}
Dental diseases are a persistent problem, not only in humans, but very often in companion animals as well. Aetiological agents of these diseases are the dental plaque bacteria. In the present study, we focused on identifying cultivable bacteria living in the dental plaque of dogs, specifically dogs suffering from the early stages of periodontal disease. Canine oral bacteria pose a risk to humans that get bitten by the dog, but they also have a zoonotic potential. Dental plaque samples were taken from five dogs of small breeds. Samples were cultured under aerobic and anaerobic conditions on several types of microbiological agars. All obtained and selected bacterial colonies were identified by PCR with universal primers for the 16S rRNA gene and the sequences of the 16S rRNA gene were compared with the sequences available in the GenBank database using BLASTn analysis. A total of 75 bacteria belonging to five phyla, predominantly to Firmicutes and Proteobacteria, were identified. The most frequent species was Pasteurella canis which was detected in all samples. In addition, representatives of the genera Actinomyces, Bacillus, Bacteroides, Corynebacterium, Frederiksenia, Fusobacterium, Haemophilus, Lactobacillus, Leucobacter, Neisseria, Ottowia, Porphyromonas, Pseudomonas, Staphylococcus, Stenotrophomonas and Streptococcus were detected in the samples. In the present study, a broad spectrum of bacteria in dental plaque samples, including canine periodontal pathogens such as Porphyromonas gulae or Porphyromonas macacae were identified. In addition, highly pathogenic bacteria, specifically Actinomyces hordeovulneris, Bacillus circulans, and Bacteroides pyogenes, which pose a serious risk to human health, were detected in samples.
\end{abstract}

Dental biofilm, oral bacteria, periodontal disease, bite wounds, dog

Dental plaque is a community of microorganisms found on the surface of teeth as a biofilm, embedded in a matrix of organic polymers of bacterial and host origin. Plaque is natural and, like the resident microbiota of all other sites in the body, contributes to the normal development of host physiology and defense (Marsh 2006). On the other hand, dental plaque (supragingival and subgingival) is an aetiological factor in dental diseases such as dental caries and periodontal diseases (Gurenlian 2007).

Periodontal disease is one of the most common and widespread inflammatory diseases in dogs, with up to $80 \%$ of animals affected (Pereira Dos Santos et al. 2019). Periodontal disease refers to a group of inflammatory processes affecting the periodontium. The periodontium contains the supporting structure of the teeth and includes the gingiva, alveolar bone, periodontal ligament and cementum (Albuquerque et al. 2012). The bacteria of dental plaque are capable of initiating the mechanisms of destruction of periodontal tissues. Inflammation of tissues surrounding the teeth progresses from an early stage (gingivitis) to an advanced stage (periodontitis). Pathological changes associated with gingivitis including 
oedema, odour, haemorrhage and exudates are reversible. Periodontitis is irreversible, leading to periodontal pocket formation, alveolar bone resorption and eventual tooth loss (Shoukry et al. 2007).

Periodontal disease in dogs is a naturally occurring disease and as such, it is more likely to have the same pathophysiological mechanisms as human periodontal disease. Histological traits of the normal and diseased periodontium are similar in humans and dogs. Moreover, several comparative studies have shown that the factors involved in canine periodontal disease, including bacterial plaque, are similar to those of humans (Albuquerque et al. 2012). Whereas the transmission of oral bacteria is also possible during normal dog-tohuman contact, potentially zoonotic and periodontopathic bacteria in the canine oral cavity can pose a problem for the oral health of humans. The canine oral bacteria involved in periodontal disease are also isolated from infected dog bite wounds in human. This is not unexpected as periodontitis is a common disease in dogs and organisms present at contaminated sites may be transmitted by the biter to the victim (Forsblom et al. 2002).

The aim of the study was to isolate and identify dental biofilm bacteria in dogs suffering from early stages of periodontal disease as a potential source of bacterial infections in humans. We focused on a group of dogs living in the same household and compared the composition of individual dental biofilms.

\section{Materials and Methods}

\section{Animals and sampling}

Dental plaque samples were taken from five dogs (aged 1.5 to 13 years) at the Clinic of Small Animals, University of Veterinary Medicine and Pharmacy in Košice. The owner filled out a questionnaire regarding the health condition, type of food, dental care and habits of each dog. The dogs sampled were two Maltese (females, neutered), one Yorkshire Terrier (female, neutered), one Jack Russell Terrier (male, neutered) and one Chihuahua cross (male, neutered) living in one household. Every dog had clinical signs of early stages of periodontal disease. The samples were taken from the buccal surfaces of upper dentes canini and dentes premolares (Plate V, Fig. 1) with a sterile syringe needle. They were immediately placed in a sterile Eppendorf tube containing $500 \mu 1$ of sterile phosphate-buffered saline and processed within 2-3 h after collection.

\section{Sample processing}

The Eppendorf tubes with samples were vortexed at maximum speed for $20 \mathrm{~s}$ (Vortex V-1 plus, Biosan, Riga, Latvia) and shook at 400 r.p.m. for 20 min (Orbital Shaker PSU-10i, Biosan) for content homogenization. Homogenized samples, specifically at a volume of $50 \mu$, were inoculated to Brain Heart Infusion broth (BHI broth; pH $7.4 \pm 0.2$, HiMedia, Mumbai, India) and deMan-Rogosa-Sharpe broth (MRS broth; pH $6.2 \pm 0.2$, Carl Roth $\mathrm{GmbH}$, Karlsruhe, Germany). After 2 days of cultivation at $37{ }^{\circ} \mathrm{C}, 50 \mu \mathrm{l}$ of BHI broth was spread onto the Brain Heart Infusion agar (BHI; $\mathrm{pH} 7.4 \pm 0.2$, HiMedia) and $50 \mu 1$ of MRS broth was spread onto the deMan-Rogosa-Sharpe agar (MRS; pH $6.4 \pm 0.2$, Carl Roth $\mathrm{GmbH}$ ). Aliquots of homogenized $50 \mu 1$ samples were spread onto the Trypticase soy agar (TSA; $\mathrm{pH} 7.2 \pm 0.1$, Carl Roth GmbH, Karlsruhe, Germany) with 5\% ram's blood and M-Enterococcus agar (ME; pH 7.2 \pm 0.2 , Becton, Dickinson and Co., Le Pont de Claix, France). Samples were cultured under aerobic and anaerobic (BBL GasPak ${ }^{\mathrm{TM}}$ Plus, Becton, Dickinson and Co., Maryland, USA) conditions at $37^{\circ} \mathrm{C}$. After 2 days of aerobic cultivation and after 3 days of anaerobic cultivation, individual solitary colonies with different morphological characteristics were selected and subcultured to obtain pure bacterial cultures. After 7 days of anaerobic cultivation, plates were again examined for the detection of black-pigmented colonies of Porphyromonas spp. The pure bacterial colonies were transferred to Eppendorf tubes containing BHI or MRS broth depending on the type of bacteria. Subsequently, glycerol (20\% v/v) was added and the isolates were stored at $-80^{\circ} \mathrm{C}$.

\section{Phenotypic determination}

Individual solitary colonies were characterized by size, colour, shape, growth form and the presence of haemolysis. They were subjected to Gram staining to verify the purity and to determine the shape, size, and arrangement of the individual bacteria. A test for catalase production was also performed using the standard protocol.

\section{DNA isolation and sequencing}

DNAzol direct (Molecular Research Center Inc., Cincinnati, USA) was used to isolate the DNA of bacteria. Pure bacterial colonies about the volume of one inoculation loop (volume $1 \mu$, BRAND GMBH $+\mathrm{CO} \mathrm{KG}$, Wertheim, Germany) were added to $50 \mu \mathrm{l}$ of DNAzol direct and heated to $95{ }^{\circ} \mathrm{C}$ for $15 \mathrm{~min}$. The $16 \mathrm{~S}$ 
ribosomal RNA (rRNA) genes from the isolates were amplified by PCR using the universal primers 27F (5'-AGAGTTTGATCMTGGCTCAG-3') and 1492R (5'-CGGYTACCTTGTTACGACTT-3'). The PCR cycling conditions comprised an initial denaturation phase of 5 min at $94^{\circ} \mathrm{C}$, followed by 30 cycles of denaturation at $94^{\circ} \mathrm{C}$ for $1 \mathrm{~min}$, annealing at $55^{\circ} \mathrm{C}$ for $1 \mathrm{~min}$ and primer extension at $72{ }^{\circ} \mathrm{C}$ for $3 \mathrm{~min}$ and finally a primer extension step at $72{ }^{\circ} \mathrm{C}$ for $10 \mathrm{~min}$. The PCR was performed in a thermal cycler (TProfesional Basic, Biometra GmbH, Göttingen, Germany), aliquot PCR products were separated by horizontal 3\% (w/v) agarose gel electrophoresis in Tris-acetate-EDTA buffer ( $\mathrm{pH} 7.8$ ) and visualized with GelRed (Biotium, Inc., Hayward, CA, USA) under ultraviolet light. The amplification products were sent for purification and sequencing using primer $1492 \mathrm{R}$ (Microsynth, Wien, Austria).

\section{Sequence processing}

The obtained chromatograms of sequences of approximately 1100 bases were edited using Geneious 8.0.5 (Biomatters, Auckland, New Zealand). All isolates were initially identified by performing database searches, comparing 16S rRNA sequences obtained from a single reading with sequences available in the GenBank using the Basic Local Alignment Search Tools, nucleotide (BLASTn) (http://www.ncbi.nlm.nih.gov/BLAST/) from the National Center for Biotechnology Information (NCBI). After identification of all isolates, the sequences of the same bacterial species isolated from one dental plaque were compared and the best quality sequence was selected. The phylogenetic tree was constructed from the alignment of the 53 selected sequences with a length of 960 bases also using the Geneious 8.0.5 program (Biomatters).

Nucleotide sequence accession numbers

The nucleotide sequences have been deposited in GenBank with accession numbers from MT492070 to MT492123.

\section{Results}

A total of 75 isolates were obtained from the plaque of 5 dogs with early stages of periodontal disease. The highest number of bacterial isolates was obtained from dog no. 1 $(\mathrm{n}=18)$ and the lowest number of bacterial isolates from dog no. $4(\mathrm{n}=10)$. The average number of isolates per dog reached 15. The predominant bacteria were Gram-negative rods, which accounted for $36 \%(\mathrm{n}=27)$. The prevalence of cultivable bacteria based on Gram staining is shown in Table 1.

Table 1. The percentage of isolates by Gram staining.

\begin{tabular}{lccc}
\hline & Rods & Cocci & Cocobacilli \\
\hline Gram-positive & $17.33 \%(\mathrm{n}=13)$ & $26.67 \%(\mathrm{n}=20)$ & $0 \%(\mathrm{n}=0)$ \\
Gram-negative & $36 \%(\mathrm{n}=27)$ & $8 \%(\mathrm{n}=6)$ & $12 \%(\mathrm{n}=9)$ \\
\hline
\end{tabular}

Of the 75 isolates sequenced, 70 were identified at the species level and the other 5 were identified at the genus level, because in the GenBank database the sequences are deposited only at the genus level. The identified isolates belong to five phyla, predominantly Firmicutes and Proteobacteria. A summary phylogenetic tree of the identified bacteria with accession numbers are shown in Fig. 2 (Plate VI).

Of the total number of isolates, 41 were isolated from media cultured under aerobic conditions. However, 34 isolates were isolated from media cultured under anaerobic conditions. The distribution of identified bacteria based on the relation to oxygen is shown in Table 2.

The widest spectrum of bacteria was isolated after the cultivation on TSA with 5\% ram's blood. Isolates belonged to these species: Actinomyces hordeovulneris, Bacteroides heparinolyticus, Bacteroides pyogenes, Corynebacterium canis, Frederiksenia canicola, Fusobacterium canifelinum, Haemophilus haemoglobinophilus, Leucobacter spp., Neisseria canis, Neisseria shayeganii, Neisseria weaveri, Ottowia spp., Pasteurella canis, Porphyromonas gulae, Porphyromonas macacae, Schaalia canis, Schaalia suimastitidis, Stenotrophomonas spp. and Streptococcus canis. A wide spectrum of bacteria was isolated 
Table 2. The distribution of isolated bacteria based on oxygen requirements.

\begin{tabular}{llll}
\hline \multicolumn{1}{c}{ Obligate aerobes } & \multicolumn{1}{c}{ Facultative anaerobes } & Microaerophiles & Obligate anaerobes \\
\hline Bacillus spp. & Actinomyces hordeovulneris & Lactobacillus animalis & Bacteroides pyogenes \\
Leucobacter spp. & Bacillus circulans & Schaalia canis & Bacteroides heparinolyticus \\
Neisseria canis & Corynebacterium canis & Schaalia suimastitidis & Fusobacterium canifelinum \\
Neisseria shayeganii & Frederiksenia canicola & & Porphyromonas gulae \\
Neisseria zoodegmatis & Haemophilus haemoglobinophilus & \\
Neisseria weaveri & Ottowia spp. & \\
Pseudomonas aeruginosa & Pasteurella canis & \\
Stenotrophomonas spp. & Staphylococcus epidermidis & \\
& Staphylococcus hominis & \\
& Staphylococcus pseudintermedius & \\
& Streptococcus canis & \\
& Streptococcus cristatus & \\
& Streptococcus gallolyticus & \\
& Streptococcus sinensis & \\
& Streptococcus suis & \\
& Streptococcus spp. & \\
\hline
\end{tabular}

after the cultivation on BHI agar, specifically the following species: Bacillus licheniformis, Bacillus circulans, Neisseria canis, Neisseria zoodegmatis, Pseudomonas aeruginosa, Staphylococcus epidermidis, Staphylococcus pseudintermedius, Streptococcus gallolyticus, Streptococcus cristatus, Streptococcus suis and Streptococcus intermedius. Staphylococcus pseudintermedius and Lactobacillus animalis were isolated from MRS agar. Only Streptococcus sinensis was isolated from ME agar. The representation of microorganisms in individual dental plaques together with anamnestic data of each dog are shown in Table 3.

\section{Discussion}

The presence and relative proportion of streptococci in the oral cavity of dogs is a longdiscussed topic. In some studies, the presence of streptococci was not detected or was detected in very low numbers; in other studies, several species were detected in the dental plaque of dogs (Harvey et al. 1995; Elliott et al. 2005; Sanguansermsri et al. 2017). In the present study, we detected Streptococcus sinensis (in 3 of $5 \mathrm{dogs}$ ), S. intermedius (in 2 of 5 dogs), S. canis (in 1 of 5 dogs), S. suis (in 1 of 5 dogs), S. gallolyticus (in 1 of 5 dogs) and $S$. cristatus (in 1 of 5 dogs). Streptococcus canis is the most common streptococcal species found in dog infections such as dermatitis, otitis externa, pneumonia, infective endocarditis and adult septicaemia. Streptococcus canis is also a zoonotic pathogen that is transferred primarily from companion animals such as dogs and cats to humans through animal bites and other mechanisms (Pinho et al. 2013; Taniyama et al. 2017). Streptococcus suis is a major swine pathogen causing meningitis, sudden death, septicaemia, polyserositis, arthritis, endocarditis, otitis and pneumonia. Streptococcus suis has also been increasingly isolated from a wide range of animal species including dogs (Muckle et al. 2014). Streptococcus gallolyticus is commonly found in the rumen flora of herbivores. It is an opportunistic pathogen causing septicaemia and endocarditis in elderly persons and is also strongly associated with colorectal cancer (Pasquereau-Kotula et al. 2018). Streptococcus sinensis was for the first time isolated from a patient with infective endocarditis (Woo et al. 2002). Later, other patients with infective endocarditis caused by $S$. sinensis were reported (Woo et al. 2004). The natural reservoir of $S$. sinensis appears 
Table 3. Anamnestic data of dogs and cultivable bacteria identified in their dental plaque.

\begin{tabular}{ll}
\hline Dog & \multicolumn{1}{c}{ Anamnesis } \\
\hline & Age, breed: 13 years, Jack Russell Terrier \\
& Sex: Male, neutered \\
& Present illnesses: Renal hypertension \\
& Prior illnesses: Babesiosis, gastritis, enteritis \\
& Current medications: None \\
& Ultrasonic teeth cleaning: Every two years \\
No. $1 \quad$ Granules for dogs: Specific CKD Heart \& Ki
\end{tabular}

No. 1 Granules for dogs: Specific CKD Heart \& Kidney Support

Canned dog food: Specific CKW Kidney Support (occasionally only)

Homemade food: No

Raw meat: No

Does he bite pet toys? Yes

Does he bite chew sticks? No

Does he bite hard objects? No

Age, breed: 7 years, Maltese

Sex: Female, neutered

Present illnesses: Food allergy with manifestations on the skin

Prior illnesses: Gastritis, enteritis

Current medications: None

Ultrasonic teeth cleaning: Every two years

No. 2 Granules for dogs: Specific CDD HY Food Allergy Management Canned dog food: Specific CDW Food Allergy Management

Homemade food: No

Raw meat: No

Does she bite pet toys? Yes

Does she bite chew sticks? Yes

Does she bite hard objects? Yes

Age, breed: 5 years, Yorkshire Terrier Corynebacterium canis

Sex: Female, neutered

Present illnesses: None

Prior illnesses: Endometritis

Current medications: None

Ultrasonic teeth cleaning: Every two years

No. 3 Granules for dogs: Specific CXD-S Adult Small Breed

Canned dog food: SPECIFIC CXW Adult All Breeds

Homemade food: No

Raw meat: No

Does she bite pet toys? Yes

Does she bite chew sticks? Yes

Does she bite hard objects? Yes

Age, breed: 9 years, Chihuahua cross

Sex: Male, neutered

Present illnesses: Epileptiform symptoms

Prior illnesses: History unknown

Current medications: None

Ultrasonic teeth cleaning: Last year

No. 4 Granules for dogs: Specific CID Digestive Support

Fusobacterium canifelinum

Haemophilus haemoglobinophilus

Neisseria weaveri

Pasteurella canis

Porphyromonas gulae

Porphyromonas macacae

Schaalia canis

Staphylococcus hominis

Staphylococcus pseudintermedius

Stenotrophomonas spp.

Streptococcus intermedius

Streptococcus cristatus

Actinomyces hordeovulneris

Bacteroides pyogenes

Ottowia spp.

Pasteurella canis

Pseudomonas aeruginosa

Streptococcus sinensis

and Specific CGD-S Senior Small Breed

Canned dog food: No

Homemade food: No

Raw meat: No

Does he bite pet toys? No

Does he bite chew sticks? No

Does he bite hard objects? No

Age, breed: 1.5 years, Maltese

Sex: Female, neutered

Present illnesses: Food allergy

Prior illnesses: History unknown

Current medications: None

Ultrasonic teeth cleaning: Not yet

No. 5 Granules for dogs: Specific CDD HY Food Allergy Management Canned dog food: Specific CDW Food Allergy Management

Homemade food: No

Raw meat: No

Does she bite pet toys? Yes

Does she bite chew sticks? Yes

Does she bite hard objects? Yes

\author{
Bacteroides heparinolyticus \\ Bacteroides pyogenes \\ Corynebacterium canis \\ Frederiksenia canicola \\ Leucobacter spp. \\ Pasteurella canis \\ Schaalia canis \\ Schaalia suimastitidis \\ Staphylococcus epidermidis \\ Staphylococcus pseudintermedius \\ Streptococcus sinensis
}


to be the oral cavity, probably the source of infection in human patients with infective endocarditis (Woo et al. 2008). Streptococcus intermedius and S. cristatus are common members of the human oral microbiota. The present study showed that $S$. sinensis, $S$. gallolyticus, $S$. intermedius and $S$. cristatus are also present in the oral cavity of dogs. Streptococcus cristatus has an antagonistic relationship with Porphyromonas gingivalis (the keystone pathogen in human periodontitis). Streptococcus cristatus is able to repress the expression of several virulence genes of $P$. gingivalis, which are involved in the production of fimbriae and gingipains (Ho et al. 2017). Observation of the negative correlation between $P$. gingivalis and $S$. cristatus distribution provides opportunities to develop therapeutics specifically against $P$. gingivalis colonization in the subgingival plaque (Wang et al. 2009).

In the study of Elliott et al. (2005), Porphyromonas, Actinomyces and Neisseria were the most frequently isolated genera from the canine dental plaque. Other genera detected in that study (Elliott et al. 2005) were Bacteroides, Fusobacterium, Corynebacterium, Haemophilus and Pasteurella, which is in accordance with our results. In the study of Pieri et al. (2018), Staphylococcus, Enterococcus and Streptococcus were the prevalent genera in the dental plaque of dogs without clinical signs of periodontal disease. Among others, they also identified the genera Bacillus, Lactobacillus, Neisseria, Pasteurella, Haemophilus and Actinomyces (Pieri et al. 2018). These genera could be associated with the health of the oral cavity of dogs. On the other hand, the results of the study of Takada and Hirasawa (2000) suggest that bacteria from the family Actinomycetaceae (Corynebacterium and Actinomyces) may play an important role in periodontitis in dogs. The bacteria associated with periodontal diseases are predominantly Gram-negative anaerobic bacteria in both humans and dogs (Lovegrove 2004; Özavci et al. 2019). In the present study, Gram staining determined $56 \%$ of negative rods, cocci, and cocobacilli, representing 42 isolates. The isolates Porphyromonas spp. were catalase-positive, which corresponds with the results of previous studies (Harvey et al. 1995; Elliott et al. 2005). Porphyromonas gulae, Porphyromonas salivosa (current name Porphyromonas macacae) and Porphyromonas denticanis are most frequently isolated from dogs with periodontal disease (Holden et al. 2017). The presence of $P$. gulae and/or $P$. macacae was detected in 3 from 5 samples of dental plaque in present study. Periodontal disease in dogs is associated with a wide range of anaerobic bacteria, including fastidious bacteria. In the present study, BHI agar and TSA with 5\% ram's blood were used to capture the widest range of bacteria. This media supports the good growth of many fastidious and non-fastidious microorganisms, including anaerobes. BBL GasPak ${ }^{\mathrm{TM}}$ Plus was used to ensure anaerobic conditions. Under these conditions, we were able to isolate obligate aerobes, facultative anaerobes, microaerophiles, as well as several obligate anaerobes. To detect other fastidious anaerobes, it is necessary to use an enriched media supplemented with L-cysteine hydrochloride, haemin and vitamin $\mathrm{K} 1$. Another way to detect fastidious, difficult-to-culture and also unculturable bacteria is to use molecular methods such as amplicon sequencing.

In the study of Özavci et al. (2019), the presence of periodontal pathogens, P. gingivalis, P. gulae, and Prevotella nigrescens, was detected by PCR. Another study using molecular biological approaches showed that the prevalence of $P$. gingivalis, Treponema denticola, Tannerella forsythia and Fusobacterium nucleatum is similar in humans and dogs (Gołyńska et al. 2017). The results of the mentioned studies (Gołyńska et al. 2017; Özavci et al. 2019) confirmed that some periodontal pathogens are common to dogs and humans. Based on these findings, the oral transmission of periodontal bacteria from dogs to humans, especially their owners, can present a risk for human oral health.

Pasteurella canis which is the most common species isolated from infected wounds after a dog bite was isolated from all samples in the present study. Other common species isolated from infected dog bite wounds detected in present study were Streptococcus intermedius, 
Staphylococcus epidermidis, Staphylococcus hominis, Neisseria weaveri, Neisseria zoodegmatis, Bacillus circulans, Fusobacterium canifelinum and Porphyromonas macacae.

The dogs included in the present study lived under the same conditions, but were fed different diets, which could affect the composition of their dental plaque. In dog no. 4, the lowest number of bacterial species was identified and Porphyromonas spp. were not detected. This may be due to the fact that this dog does not eat soft diets. There is reasonable evidence that soft diets are associated with increased frequency and severity of periodontal disease, and that harder foods requiring vigorous prehension and mastication are preferable for dogs (Gawor et al. 2006).

In conclusion, our results confirmed the presence of several genera often detected in studies focused on canine dental bacterial biofilm composition, especially Actinomyces, Bacteriodes, Corynebacterium, Fusobacterium, Haemophilus, Neisseria or Porphyromonas. In addition, other genera such as Leucobacter, Ottowia, Pseudomonas and Stenotrophomonas were identified. Pasteurella canis, the most commonly isolated bacterium from human bite wounds, was detected in all canine samples. The presence of pathogenic bacteria including Bacillus circulans, Actinomyces hordeovulneris, Bacteroides pyogenes was also detected in the canine dental plaque samples. Our findings confirmed that bacteria in the canine dental plaque present a serious risk of bacterial infection not only in bite wounds but also in oral transmission. All bacteria present high health risks after transmission to the bloodstream, especially in the case of highly pathogenic bacteria.

\section{Conflict of interest}

The authors declare no conflict of interest.

\section{Acknowledgements}

This publication was supported by the Scientific Grant Agency of the Ministry of Education of the Slovak Republic under the contract no. VEGA 1/0788/19 and the project implementation: Medical University Science Park in Košice (MediPark, Košice) ITMS 26220220185 supported by the Operational Programme Research and Development, funded by the ERDF.

\section{References}

Albuquerque C, Morinha F, Requicha J, Martins T, Dias I, Guedes-Pinto H, Bastos E, Viegas C 2012: Canine periodontitis: the dog as an important model for periodontal studies. Vet J 191: 299-305

Elliott DR, Wilson M, Buckley CM, Spratt DA 2005: Cultivable oral microbiota of domestic dogs. J Clin Microbiol 43: 5470-5476

Forsblom B, Sarkiala-Kessel E, Kanervo A, Väisänen ML, Helander IM, Jousimies-Somer H 2002: Characterisation of aerobic gram-negative bacteria from subgingival sites of dogs--potential bite wound pathogens. J Med Microbiol 51: 207-220

Gawor JP, Reiter AM, Jodkowska K, Kurski G, Wojtacki MP, Kurek A 2006: Influence of diet on oral health in cats and dogs. J Nutr 136: 2021-2023

Gołyńska M, Polkowska I, Bartoszcze-Tomaszewska M, Sobczyńska-Rak A, Matuszewski Ł 2017: Molecularlevel evaluation of selected periodontal pathogens from subgingival regions in canines and humans with periodontal disease. J Vet Sci 18: 51-58

Gurenlian JR 2007: The role of dental plaque biofilm in oral health. J Dent Hyg 81: 1-11

Harvey CE, Thornsberry C, Miller BR 1995: Subgingival bacteria--comparison of culture results in dogs and cats with gingivitis. J Vet Dent 12: 147-150

Ho MH, Lamont RJ, Xie H 2017: Identification of Streptococcus cristatus peptides that repress expression of virulence genes in Porphyromonas gingivalis. Sci Rep 7: 1413

Holden JA, O’Brien-Simpson NM, Lenzo JC, Orth RKH, Mansell A, Reynolds EC 2017: Porphyromonas gulae activates unprimed and gamma interferon-primed macrophages via the pattern recognition receptors Toll-Like Receptor 2 (TLR2), TLR4, and NOD2. Infect Immun 85: e00282-17

Lovegrove JM 2004: Dental plaque revisited: bacteria associated with periodontal disease. J N Z Soc Periodontol 87: 7-21

Marsh PD 2006: Dental plaque as a biofilm and a microbial community - implications for health and disease. BMC Oral Health 6: S14 
Muckle A, López A, Gottschalk M, López-Méndez C, Giles J, Lund L, Saab M 2014: Isolation of Streptococcus suis from 2 lambs with a history of lameness. Can Vet J 55: 946-949

Özavci V, Erbas G, Parin U, Yüksel HT, Kirkan Ş 2019: Molecular detection of feline and canine periodontal pathogens. Vet Anim Sci 8: 100069

Pasquereau-Kotula E, Martins M, Aymeric L, Dramsi S 2018: Significance of Streptococcus gallolyticus subsp. gallolyticus association with colorectal cancer. Front Microbiol 9: 614

Pereira Dos Santos JD, Cunha E, Nunes T, Tavares L, Oliveira M 2019: Relation between periodontal disease and systemic diseases in dogs. Res Vet Sci 125: 136-140

Pieri FA, Silva VD, Junior AS, Moreira MA 2018: Cultivable microbiota in Mitis Salivarius agar from dental plaque of dogs. Anim Vet Sci 6: 21-26

Pinho MD, Matos SC, Pomba C, Lübke-Becker A, Wieler LH, Preziuso S, Melo-Cristino J, Ramirez M 2013: Multilocus sequence analysis of Streptococcus canis confirms the zoonotic origin of human infections and reveals genetic exchange with Streptococcus dysgalactiae subsp. equisimilis. J Clin Microbiol 51: 1099-1109

Sanguansermsri P, Chairatvit K, Roytrakul S, Doungudomdacha S, Surarit R 2017: Exploring difference in subgingival microbial communities in dog and human periodontal diseases using DGGE technique. TJVM 47: $7-14$

Shoukry M, Ben Ali L, Abdel Naby M, Soliman A 2007: Repair of experimental plaque-induced periodontal disease in dogs. J Vet Dent 24: 152-165

Takada K, Hirasawa M 2000: Expression of trypsin-like activity by the genera Corynebacterium and Actinomyces in canine periodontitis. J Med Microbiol 49: 621-625

Taniyama D, Abe Y, Sakai T, Kikuchi T, Takahashi T 2017: Human case of bacteremia caused by Streptococcus canis sequence type 9 harboring the $\operatorname{scm}$ gene. IDCases 7: 48-52

Wang BY, Wu J, Lamont RJ, Lin X, Xie H 2009: Negative correlation of distributions of Streptococcus cristatus and Porphyromonas gingivalis in subgingival plaque. J Clin Microbiol 47: 3902-3906

Woo PC, Tam DM, Leung KW, Lau SK, Teng JL, Wong MK, Yuen KY 2002: Streptococcus sinensis sp. nov., a novel species isolated from a patient with infective endocarditis. J Clin Microbiol 40: 805-810

Woo PC, Teng JL, Leung KW, Lau SK, Tse H, Wong BH, Yuen KY 2004: Streptococcus sinensis may react with Lancefield group F antiserum. J Med Microbiol 53: 1083-1088

Woo PC, Teng JL, Tsang SN, Tse CW, Lau SK, Yuen KY 2008: The oral cavity as a natural reservoir for Streptococcus sinensis. Clin Microbiol Infect 14: 1075-1079 
Plate V

Mad'ar M. et al.: Cultivable ... pp. 171-178

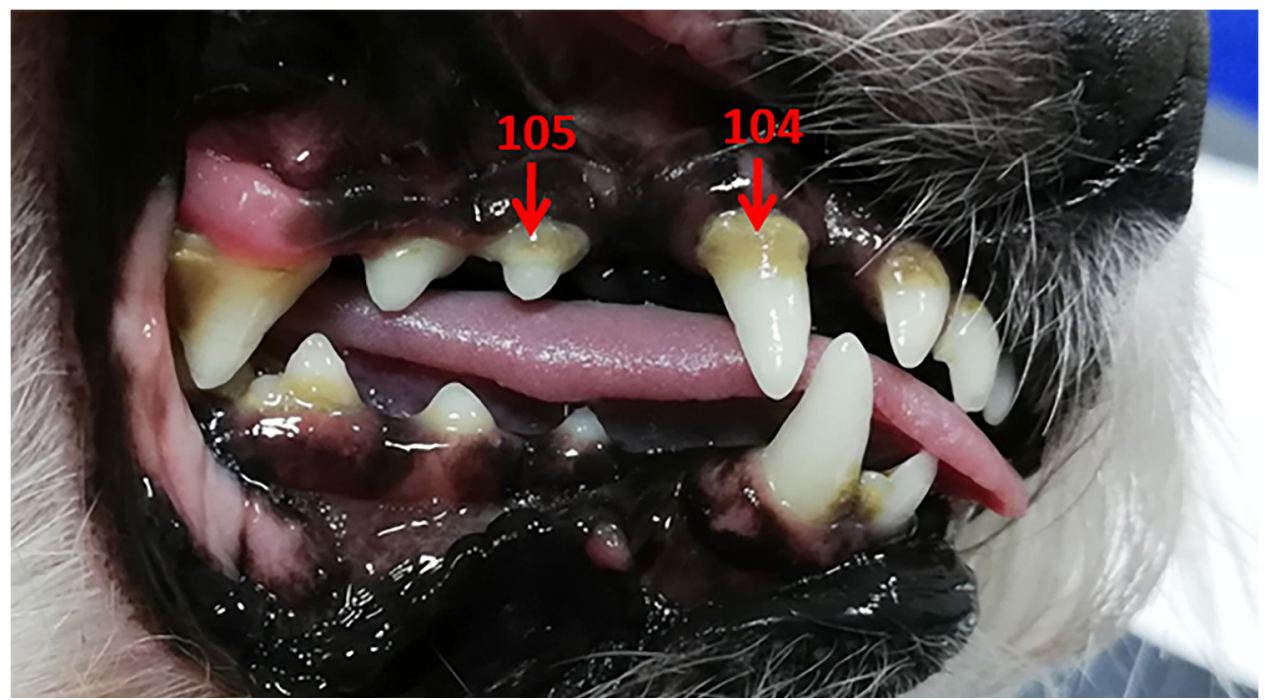

Fig. 1. Places of sample collection

104 - maxillary right dens caninus; 105 - maxillary right first premolar 


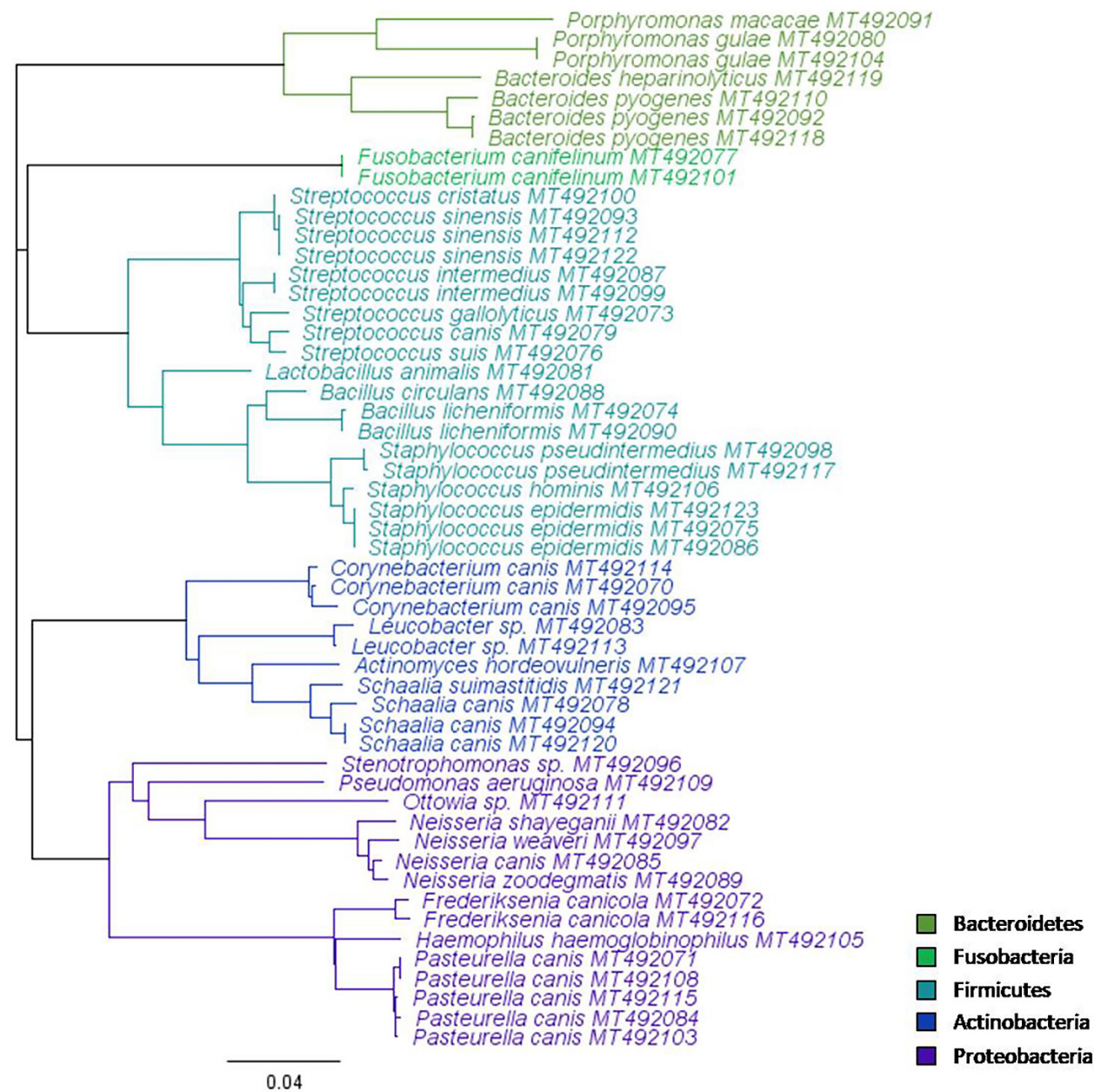

Fig. 2. Phylogenetic tree of the identified bacteria with the best quality sequence.

Sequences are identified to the species or genus level. The name of each isolate is followed by the GenBank accession number. The tree is based on an alignment of 960 bases and was constructed with Geneious version 8.0 (Biomatters, available from http://www.geneious.com). 